It was not found possible to use good solvents for dichloro-ethylsulfide as absorbing solutions and then determine the strength by our method, for the reason that the only suitable solvents (alcohol and acetic acid) reduced the selenious acid to some extent.

The selenious acid reagent is not specific to mustard gas. Arsine and the substituted arsines and several other toxic gases react with it vigorously.

Selenious acid may have a possible use as an indicator in oxidationreduction reactions.

We wish to express our appreciation of the work of A. E. Plumb, who made a great many of the determinations.

\title{
Summary.
}

A solution of selenious acid in $I: I$ sulfuric acid is reduced by traces of dichloro-ethylsulfide giving an orange-red suspension of selenium. About $0.005 \mathrm{mg}$. of dichloro-ethylsulfide can be detected in this manner. A nephelometric procedure for determining amounts of the substance between $0.1 \mathrm{mg}$. and $0.0 \mathrm{mg}$. with a maximum error of $0.005 \mathrm{mg}$. has been developed.

WashINGTON, $\mathfrak{D}, \mathrm{C}$.

[Contribution from the Research Laboratory of the General Electric ComPANY.]

\section{THE OCTET THEORY OF VALENCE AND ITS APPLICATIONS WITH SPECIAL REFERENCE TO ORGANIC NITROGEN COMPOUNDS.}

\section{By IRVING LANGMUIR.}

Received July 7, 1919.

The octet theory of valence ${ }^{1}$ leads to structural formulas for organic compounds which are identical with those given by the ordinary valence theory whenever we can assume a valence of 4 for carbon, 3 for nitrogen, 2 for oxygen and one for hydrogen and chlorine. This follows from the following reasoning:

Let us represent by $E$ the number of electrons in the shell of an atom. For the elements of the two short periods $E$ is equal to the ordinal number of the group in the periodic system. Thus for hydrogen and sodium $E=\mathrm{I}$, for carbon $E=4$, for nitrogen and phosphorus $E=5$, for oxygetn and sulfur $E=6$ and for chlorine $E=7$.

We represent by $e$ the total number of electrons in the shells of the atoms that combine to form a molecule. Let $n$ be the number of octets formed

1 G. N. Lewis, This Journal, 38, 762 (I9I6); Langmuir, Ibid., 4I, 868, 1543 (x919); and Proc. Nat. Acad. Sci., 5, 252 (1919). 
from these electrons, and let $p$ be the number of pairs of electrons which the octets share with one another. Since every pair of electrons thus shared reduces by two the number of electrons required to form the molecule, we are led to the "octet equation."

$$
p=1 / 2(8 n-e) \text {. }
$$

Each pair of electrons held in common by two octets corresponds to a covalence bond. In addition to bonds given by this equation there are those corresponding to pairs of electrons which hold hydrogen nuclei. Thus if $H$ represents the number of hydrogen atoms in a molecule, the total number of covalence bonds $v$ in the molecule is

$$
v=p+H \text {. }
$$

Let $C, N, X$ and $C l$ represent, respectively, the numbers of carbon, nitrogen, oxygen and chlorine atoms in the molecule. Equation $I$ thus gives

$$
2 p=8(C+N+X+C l)-\left(4 C+5 N+6 X+{ }_{7} C l+H\right) \text {. }
$$

Simplifying and substituting in (2) we obtain

$$
2 v={ }_{4} C+3 N+2 X+C l+H .
$$

We are also led to this equation if we calculate, by the ordinary valence theory, the total number of valence bonds in a molecule, assuming the valences to be, respectively, 4, 3, 2 and I for carbon, nitrogen, oxygen and chlorine. These valences are Abegg's countervalences and are each equal to $8-E$. The factor 2 in the first term of Equation 4 results from the fact that in adding ${ }_{4} C,{ }_{3} N$, etc., we count each bond twice.

It is thus proved that any formula written in accordance with the ordinary valence theory will be in agreement with the octet theory, if we take the valence of hydrogen as unity and that of each other atom as $8-E$. On the other hand, it is apparent that with valences other than $8-E$, the ordinary valence theory leads to results different from those of the octet theory. It is the object of this paper to deal especially with cases of this kind.

As the first example, let us consider sodium chloride. For sodium $8-E$, is 7 while the ordinary valence theory assumes a valence of unity. Applying the octet theory to sodium chloride, we place $e=1+7=8$. The chlorine atom forms an octet, but we cannot reasonably assume that the sodium takes up 7 more electrons to complete an octet. Therefore, $n=\mathrm{I}$ and Equation $\mathrm{I}$ gives $p=0$. The covalences of chlorine and sodium in this compound are thus each zero. But since $n=\mathrm{I}$ the chlorine atom must have a complete octet and must, therefore, be negatively charged. Similarly the sodium atom, having lost its extra electron, will be positively charged. The atoms are held together by electrostatic forces even without sharing electrons. This result is eminently satisfactory, for it explains at once why $\mathrm{X}$-ray crystal analysis gives no evidence of molecules of sodium clloride, and why salt is ionized in water solutions and in the molten 
state. This theory, however, does not indicate that sodium and chlorine ions should never be held together in pairs. The vapor of sodium chloride and solutions in non-ionizing solvents may well contain such pairs or molecules held together by the attraction of the ions, but even in this case the covalence would be zero. This conception seems more useful than one in which the valence is taken as the number of electrons transferred from one atom to the other. In the cases just considered it is so obvious that the two ions will tend to be drawn together by their electrostatic attraction, that we hardly need to use the conception of valence to describe the attraction.

This theory is in full accord with the theory of S. R. Milner ${ }^{1}$ in which he argues that the ions in solutions of strong electrolytes are never associated into molecules, but that pairs of ions which are temporarily near together may act as if bound together electrostatically. This theory explains the failure of Ostwald's dilution law for strong electrolytes, and is quantitatively in agreement with the best experimental data on the conductivity of electrolytes. Almost identical theories of strong electrolytes have been developed recently by Bjerrum ${ }^{2}$ and by Ghosh. ${ }^{3}$

As a second example let us consider the structure of ammonium chloride, a typical case in which the nitrogen is certainly not tervalent. Here $e=16, n=2$ and we obtain by Equation $1, p=0$. This means that the nitrogen and the chlorine octets do not hold electrons in common. If we consider that the 4 hydrogen nuclei are at first detached from the nitrogen and chlorine octets, then the nitrogen atom would have an excess of 3 negative charges, and the chlorine one negative charge. The hydrogen nuclei can distribute themselves between the two octets in such a way as to render them electrically neutral if 3 hydrogen nuclei are bound by 3 pairs of electrons in the nitrogen octet, while the fourth hydrogen nucleus goes to the chlorine. This gives a mixture of ammonia and hydrochloric acid gases and corresponds with the facts observed at high temperatures. On the other hand, since the more electronegative chlorine has the lesser tendency to share its electrons, the 4 hydrogen nuclei can attach themselves to the 4 pairs of electrons of the nitrogen octet forming a very symmetrical positive ion, while the chlorine remains as a negative ion. The ammonium ion and the chlorine ion thus have covalences of zero, but that of the nitrogen atom is 4 . The structure of the ammonium ion is thus exactly like that of the methane molecule except for the difference in the charge on the nucleus. The relationship between the ammonium ion and methane is described by the term isosterism and this conception proves of value in correlating the similarity between the potassium and

a Phil. Mag., 35, 214, 354 (1918).

2. Elektrochem., 24, 32x (1918).

s J. Chem. Soc. Trans., 113, 449, 627 (x918) 
the ammonium ions with that between argon and methane. ${ }^{1}$ The same kind of reasoning can be used to explain the fact that the ammonium ion resembles the potassium ion more closely than it does the sodium ion. The sodium ion has a structure like the neon atom, but neon differs from methane much more than argon does, so we should expect the sodium ion to show similar differences from the ammonium ion. The low boiling points of argon and methane show that the external field around these molecules (or atoms) must be very small. There should then be no strong forces around the potassium and ammonium ions except those due to the electrostatie charge. The markedly different properties of the silver ion proves that in other cases there are forces of a different kind which are of importance in determining the properties. The silver ion is related to the palladium atom as the potassium ion is to argon, and the great difference between argon and palladium corresponds to the difference between the potassium and silver ions.

From the examples just cited it is cleat that the octet theory applies satisfactorily even when the valence of atoms is not equal to $8-E$. In these cases structural formulas are obtained quite different from those the ordinary valence theory of organic chemistry, but they are in perfect agreement with the properties of the compounds. It is true that Werner's theory leads to the correct constitution of ammonium compounds, but only by making a large number of assumptions of a quite different kind from those on which the organic structural formulas are based. The particular merit of the octet theory is that it gives a single simple theory which includes the extreme types of compounds represented by organic compounds, the alkali halides, and so-called complex compounds.

The octet theory as expressed by Equation I is, of course, not capable of showing distinctions between compounds of two elements which have the same number of electrons in their shells, as for example between nitrogen and phosphorus. However, as was pointed out in the previous paper, the theory can be supplemented by certain more or less empirical rules regarding the covalence of the various elements. The general study of compounds by the octet theory has shown the following covalences:

The inert gases, alkali and alkaline earth metals, zero. Carbon, 4 in all ordinary organic compounds, but in some cases the covalence may be 3 as for example in calcium carbide, triphenylmethyl sodium and many other triphenylmethyl derivatives. Nitrogen, usually 3 , but 4 in most compounds where quinquevalent nitrogen has been assumed, and 2 in a few compounds. Oxygen, I, 2 or sometimes 3 (in peroxides). Fluorine, zero or $\mathrm{x}$. Phosphorus, usually 4 , sometimes 3 and rarely zero. Sulfur and chlorine may have any values from zero to 4 .

According to the ordinary theory the valences of an element usually

1 This Journal, 4I, I547 (r9i9). 
increase by steps of two, thus nitrogen and phosphorus are supposed to have valences of 3 and 5 ; sulfur, 2, 4 and 6 ; chlorine, $1,3,5$ and 7 , etc. According to the octet theory, however, where the covalence is variable, its value increases by steps of one. The doubie steps of the ordinary theory are due to the fact that the total number of electrons, $e$, must be an even number according to Equation 2, so that where univalent elements are added two atoms must be added at a time. It is well known that oxygen atoms are added singly as in $\mathrm{HNO}_{2}, \mathrm{HNO}_{3}, \mathrm{HClO}, \mathrm{HClO}_{2}, \mathrm{HClO}_{3}$ and $\mathrm{HClO}_{4}$. The ordinary theory assumes that these are held by double bonds, while the octet theory shows that they are held by single pairs of electrons.

\section{Tercovalent Nitrogen.}

It was shown by Equation 4 that the structural formulas written according to the ordinary theory are always in accord with the octet theory when the valence of nitrogen is 3 . Let us see, however, what light the octet theory throws on the stereoisomerism of nitrogen compounds.

In the previous paper reasons were given for believing that when the 8 electrons in an octet are not shared by other atoms they are normally arranged at the corners of a cube. A pair of electrons held in common by two atoms, however, acts as though it were located at a point. When an atom has a covalence of 4 the 8 electrons in its octet are, therefore, drawn together into 4 pairs which arrange themselves symmetrically at

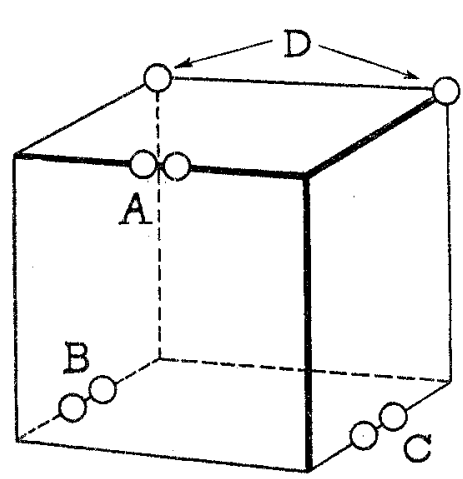

Fig. I. the corners of a regular tetrahedron. As Lewis already pointed out, stereoisomerism of carbon compounds is thus fully explained. The same reasoning applies to atoms of nitrogen, phosphorus, sulfur, etc., whenever these atoms are quadricovalent, so that stereoisomers are obtained under the same structural conditions as with carbon compounds. The experimental facts are in full accord with this conclusion.

When only 3 pairs of electrons in an octet are shared we should expect an arrangement like that shown diagrammatically in Fig. $x$.

The 3 pairs of electrons, A, B and C, which correspond to the 3 covalence bonds are arranged in space approximately like 3 of the corners of a regular tetrahedron. The fourth corner corresponds to the unshared pair, D. This arrangement of the 3 bonds in space agrees exactly with Hantzsch and Werner's ${ }^{1}$ theory which has so well explained the isomerism of aldoximes, ketoximes, hydrazones, osazones, diazo compounds, etc. Neogi's

$$
\text { I Ber., 23, I I (1890). }
$$


recent theory ${ }^{1}$ assumes an identical arrangement of the valence bonds. For a full discussion of the evidence in favor of this arrangement Neogi's paper should be consulted.

The absence of isomers among amines containing 3 different groups is not inconsistent with this theory, for it can be satisfactorily explained by a mobility of the groups caused by the presence of the unshared pair of electrons at $D$. Thus, in a compound $N R_{1} R_{2} R_{3}$ each of the 3 alkyl groups holds a pair of electrons in common with the nitrogen atom, but the nitrogen atom has another pair of electrons (D in Fig. I) which is not shared. We may assume that the alkyl groups are able to shift into the unoccupied position while the pair of electrons has mobility enough to allow it to occupy the position just vacated by the alkyl group. This could occur without the alkyl radical becoming detached from the nitrogen atom at any time. A similar mobility would not exist if all 4 pairs of electrons were shared. Stereoisomers among compounds of tervalent nitrogen are observed only when the nitrogen is held by a double bond, that is, when two of its pairs of electrons, as for example $B$ and $C$ in Fig. I, are shared by a single other atom. Such a behavior seems quite reasonable on the basis of this structure. With the double covalence bond it is natural that new constraints should be called into existence in the atom and that the mobility of its other electrons should be decreased.

Quadricovalent Nitrogen, Etc.

The structure of tetramethyl ammonium hydroxide, $\left(\mathrm{CH}_{3}\right)_{4} \mathrm{NOH}$ is found by placing $e=40 ; n=6$ which gives $p=4$. The nitrogen is thus quadricovalent (as in the ammonium ion) and the hydroxyl ion exists as a negative ion which does not share electrons with the ammonium radical. It is thus completely ionized in solution and is, therefore, a strong base. Of course any other negative ion can take the place of the hydroxyl ion.

In applying the octet theory to the structure of organic compounds it is usually convenient to consider the different parts of molecules separately. We can accomplish this by letting $R, R^{\prime}$, etc., represent univalent radicals and assuming that for each radical $E=7$ and $n=\mathrm{I}$. For example, the radical $\mathrm{C}_{3} \mathrm{H}_{7}$ in a molectle really contributes 19 electrons to $e$ and 3 octets to $n$. If we replace this by $\mathrm{R}$ we add only 7 electrons to $e$ instead of 19 and one octet to $n$ instead of 3. This decreases $n$ in Equation 2 by 2 units and decreases $e$ by 12 units. The result is that the value of $p$ found by the equation is decreased by 2 units, $i, e, 1 / 2(\mathrm{I} 6-\mathrm{I} 2)$. But since 2 pairs of electrons must be shared between octets in $\mathrm{C}_{3} \mathrm{H}_{7}$ while none are needed for $R$, it is evident that we have not affected the value of $p$ for the remainder of the molecule by substituting $\mathrm{R}$ for $\mathrm{C}_{3} \mathrm{H}_{7}$. This reasoning will be found to hold whenever $R$ represents any univalent radical. For a divalent radical we can place $E=6, n=I$.

1 THIS JOURNAL, 4I, 622 ( $19 \mathrm{Ig})$, 
According to this theory the substituted ammonium compounds should show isomerism exactly like that of carbon compounds, since the nitrogen is quadricovalent. This is in full accord with Werner's theory and with all the experimental data. Neogi ${ }^{1}$ raises an objection to Werner's theory on the grounds that the latter "has postulated the existence of as many as three kinds of valence, viz., (I) principal, (2) subsidiary valences of nitrogen, and (3) residual valence of the ammonium group." It is seen that the present theory reaches a conclusion essentially similar to Werner's without making any additional assumptions regarding valence. The octet theory thus removes the objections that have been raised against Werner's theory. Neogi's own assumption of a tetrahedral nitrogen atom is, of course, in full agreement with the octet theory, but his assumption of a fifth bond "attached to the nitrogen by principal valences, though four of them are in the inner zone and the fifth is in the outer zone" is entirely unnecessary and introduces needless complication. Neogi states that "there is no escape from acknowledging the pentavalence of nitrogen as in the amine-oxides $O: N_{1} R_{2} R_{8}$ " The octet theory gives for the amine-oxides $e=3^{2}, n=5$ and $p=4$. Thus the nitrogen is quadricovalent, and it is entirely unnecessary to assume quinquevalent nitrogen. The octet theory thus greatly simplifies our conception of the structure of compounds of this type. The amine oxides should, of course, exist in two optically isomeric forms whenever the 3 substituted radicals are different, just as in other cases of quadricovalent nitrogen. Neogi by arbitrary assumptions explains the isomerism of these oxides, but states that none of the other theories of the stereoisomerism of nitrogen compound is able to do so.

The isomeric compounds $\left(\mathrm{Me}_{3} \mathrm{NOR}\right) \mathrm{OR}_{1}$ and $\left(\mathrm{Me}_{3} \mathrm{NOR}_{1}\right) \mathrm{OR}$ prepared by Meisenheimer ${ }^{2}$ are satisfactorily explained by the octet theory. One of the OR radicals exists as a negative ion as in the alcoholates, while the other shares a pair of electrons with the nitrogen atom.

One fundamental objection to Neogi's theory is that it gives no adequate reason why only radicals capable of forming negative ions can be held by the fifth bond. ${ }^{3}$

The octet theory throws a great deal of light on the properties of ammonium compounds. Since in typical ammonium salts the $\mathrm{NH}_{4}$ group exists as an ion, the physical properties should resemble potassium salts for the reasons already given. The close relationship between the solubilities, crystalline forms, etc., of ammonium and potassium salts is in

1 Loc. cit., p. 623.

${ }^{2}$ Ann., 397, 273 (1913).

3 The absence of optical isomers of the substituted pysidinium and quinolinium compounds referred to by Neogi on p. 638 of his paper is undoubtedly to be explained by an effect in the pyridine ring similar to that which prevents the occurrence of two isomers of 0 -dichlorobenzene such as might be expected from the Kekule formula. 
fact very striking, but in certain other respects such as vapor pressures, melting points, etc., there are often striking differences. Ammonium chloride sublimes without melting at about $340^{\circ}$ at atmospheric pressure, while the corresponding potassium salt melts at $772^{\circ}$, and boils only at very much higher temperatures. It is probable that this difference is due to the dissociation into ammonia and hydrogen chloride although this simple assinaption does not wholly account for the facts since perfectly dry ammonium chloride is said to vaporize without dissociation. However, the total vapor pressure at a given temperature has been found to be the same whether or not the vapor is dissociated. ${ }^{1}$ This seemingly incomprehensible result probably indicates that something closely related to dissociation does occur even when the vapor density corresponds to that of ammonium chloride, and that in any case the so-called "undissociated" vapor probably has molecules of a quite different structure from those that would be obtained from potassium chloride. It would seem that a study of the dielectric constants of the "dissociated" and "undissociated" vapors and possibly their electric conductivities or ionizing potentials might clear up these puzzling questions.

The failure to obtain the ammonium halides in the molten condition is what is to be expected by the octet theory. ${ }^{2}$ There are, however, other ammonium salts which melt at rather low temperatures compared to the corresponding potassium salts. As examples let us consider ammonium nitrate which melts at $166^{\circ}$ while potassium nitrate melts at $337^{\circ}$, and ammonium trinitride, $\mathrm{NH}_{4} \mathrm{~N}_{3}$, which melts at $1 \mathrm{IO}^{\circ}$.

Applying the octet equation to ammonium nitrate we find $p=4$. This leads to the following possible arrangements:
(a) $\mathrm{NH}_{4}+\left[\begin{array}{c}\mathrm{O} \\ \mathrm{O}-\mathrm{N}=\mathrm{O}\end{array}\right]^{-}$;
(b) $\mathrm{NH}_{3}+\mathrm{O}=\mathrm{N}-\mathrm{OH}$ $\mathrm{O}$<smiles>[NH3+]O[N+](=O)[O-]</smiles>
or
(d)

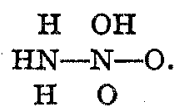

${ }^{1}$ Abegg, Z. physik. Chem., 6x, 455 (1908), and subsequent papers by van Laar, Wegscheider and Scheffer.

${ }^{2}$ With the assistance of Miss Katharine Blodgett, I have found that pure ammonium chloride, sealed up in a small, heavy-walled Pyrex glass tube and heated, melts to a clear liquid at about $550^{\circ}$. By plotting the logarithms of the vapor pressures, measured by Smith and Calvert, Trus Journal, 36, 1373 (I9I4), against the reciprocals of the corresponding absolute temperatures a straight line was obtained. Extrapolation along this line gives the vapor pressure of ammonium chloride at $550^{\circ}$ as 66 atmospheres. The ratio between the melting point of ammonium chloride $\left(823^{\circ} \mathrm{K}\right.$.) and that of potassium chloride $\left(1045^{\circ} \mathrm{K}\right.$.) is not greatly different from the ratio between the melting point of ammonium hydrogen sulfate $\left(4 \mathrm{I} 3^{\circ} \mathrm{K}\right.$.) and that of potassium hydrogen sulfate $\left(473^{\circ}\right.$ K.). The lower melting point of the ammonium salts and their somewhat grtater solubilities are probably to be accounted for by the tetrahedral shape of the ammonium ion (like that of the methane molecule) as compared to the cubical shape of the potassium ion. 
Structure $(a)$ corresponds to the salt type; this should have a melting point comparable with that of potassium nitrate.

Structure (b) corresponds to a possible dissociation into nitric acid and ammonia.

Structures $(c)$ and $(d)$ suggested by the octet theory are quite inconsistent with the ordinary valence theory. The melting of anhydrous ammonium nitrate perhaps depends, however, upon the formation of one or both of these tautomeric products.

Upon heating, (c) should give $2 \mathrm{H}_{2} \mathrm{O}+\mathrm{N}=\mathrm{O}=\mathrm{N}$, while $(d)$ should give $2 \mathrm{H}_{2} \mathrm{O}+\mathrm{N}=\mathrm{N}=\mathrm{O}$. These constitutions thus explain the formation of nitrous oxide.

We shall see from data to be given further on that there is good evidence that nitrous oxide is represented by the structure $\mathrm{N}=\mathrm{N}=\mathrm{O}$. Therefore, molten ammonium nitrate probably contains some molecules having the structure

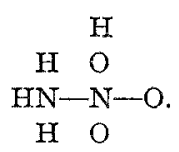

It is to be expected that molecules of this type have relatively weak external fields of force as compared to $\mathrm{NH}_{4}+\mathrm{NO}_{3}^{-}$.

In a similar way the melting of $\mathrm{NH}_{4} \mathrm{~N}_{3}$ at $\mathrm{IIO}^{\circ}$ is, perhaps, to be ascribed to the formation of molecules of the structure

$$
\begin{aligned}
& \mathrm{H} H \\
& \mathrm{H}-\mathrm{N}-\mathrm{N}
\end{aligned}
$$

According to the above hypothesis the difference between the melting points of the ammonium salts and the corresponding potassium salts is dependent on the presence of double bonds in the anion of the acid. Ammonium sulfate has an anion in which there are no double bonds, and the central atom has a covalence of 4 . It is, therefore, not possible for the ammonia group to attach itself to the sulfate ion in the same manner as with the nitrate ion. Therefore, we should expect ammonium sulfate to have a melting point only a little lower than that of potassium sulfate $\left(\mathrm{IO}_{7}{ }^{\circ}\right)$. Now most tables of physical constants and handbooks like Abegg's give the melting point of ammonium sulfate as $140^{\circ}$ These statements seemed so radically inconsistent with the octet theory that I was led to test the data by experiment. It was found that ammonium sulfate remained practically unchanged until heated to about $280^{\circ}$ and it then decomposed, giving off ammonia and yielding fused ammonium hydrogen sulfate. Subsequently I found that $\mathrm{Reik}^{1}$ had already observed, that ammonium sulfate decomposes without melting, and that it is the acid sulfate $\mathrm{NH}_{4} \mathrm{HSO}_{4}$

1 Monatsh., 23, 1033 (1902). 
which melts at $I 40^{\circ}$. This is not greatly different from the melting point of potassium hydrogen sulfate, so that the low melting point is not inconsistent with the octet theory. The fused ammonium hydrogen sulfate is a very good electrolytic conductor and probably consists wholly of ions of $\mathrm{N}_{4}{ }_{4}{ }^{+}$and $\mathrm{HSO}_{4}{ }^{-}$.

The relatively low melting point of ammonium nitrate and the infusibility of the sulfate thus support the conclusion from the octet theory that the nitrate ion has a double bond, while in the sulfate ion the 4 oxygen atoms are held to the sulfur by single bonds. The ordinary valence theory on the other hand is not capable of showing such a distinction, for it gives double bonds for both anions.

Ammonium formate, $\mathrm{NH}_{4} \mathrm{CHO}_{2}$, melts at $I 5_{5}^{\circ}$, and at $\mathrm{I} 80^{\circ}$ decomposes into formamide and water. Although this melting point is not greatly below that of the potassium salt $\left(\mathrm{I}_{5} 0^{\circ}\right)$ it is possible that the melt contains a proportion of molecules having the structure

$$
\begin{array}{cl} 
& \mathrm{H} \\
\mathrm{H} & \mathrm{O} \\
\mathrm{HN}-\mathrm{C}-\mathrm{O} \\
\mathrm{H} & \mathrm{H}
\end{array}
$$

in which one of the oxygen atoms is unicovalent. It is apparent that water can be readily split off from this molecule yielding formamide, $\mathrm{H}_{2} \mathrm{NCHO}$.

Schlenk and Holtz ${ }^{1}$ have prepared a compound, triphenylmethyltetramethy] ammonium, in which 5 hydrocarbon residues are supposed to be attached to a single nitrogen atom, and which would thus furnish direct evidence of quinquevalent nitrogen. The compound is described as forming a "red shimmering crystalline mass with blue metallic luster," which is immediately decomposed by water yielding triphenylmethane and tetramethyl ammonium hydroxide. It is formed by the action of triphenylmethyl sodium on dry tetramethyl ammonium chloride in solution in ether.

Applying the octet theory to triphenylmethyl sodium by placing $e=26, n=4$, we find $p=3$. Each of the 3 phenyl radicals shares a pair of electrons with the carbon atom, but the sodium atom shares no electrons with the organic radical. The constitution is, therefore,

$$
\mathrm{Na}+\left(\mathrm{CPh}_{3}\right)^{-} \text {. }
$$

The central carbon atom has a covalence of 3 , but has its complete octet. This formula is in full accord with the unusual properties of this substance and explains, for example, its strong color and its reaction with ammonia to form triphenylmethane and sodium amide, $\mathrm{Na}^{+} \mathrm{NH}_{2}{ }^{-}$. It is thus evident that the triphenylmethyltetramethyl ammonium which is derived from the triphenylmethyl sodium should have the constitution

\footnotetext{
1 Ber. 49,603 (1916).
} 


$$
\left[\mathrm{N}\left(\mathrm{CH}_{3}\right)_{4}\right]+\left[\mathrm{CPh}_{3}\right]^{-} \text {. }
$$

This substance should thus be a salt whose ions are held together by electrostatic attraction. The covalence of the nitrogen is 4 while that of the central carbon atom in the anion is 3 . It is stated by Schlenk and Holtz that a solution of this substance in pyridine conducts the electric current, so there can be no question of its salt-like character.

"The structure<smiles>N=C1NO1</smiles>

has been proposed for carbamide by E. A. Werner, ${ }^{1}$ while W. A. Noyes and R. S. Potter, ${ }^{2}$ in accordance with the views of others, have assumed the formula $(a)$<smiles>[R]1CO1</smiles>

for certain amino acids. In the case of compounds of this second type the octet theory gives $p=5$ which makes it impossible to place a covalence bond between the oxygen and the nitrogen. However, the formula may be written: (b)<smiles></smiles>

in which it is evident that the normally electrically neutral ammonia must become positively charged when it shares a pair of electrons with the carbon atom of the $\mathrm{R}$ group. Similarly the oxygen atom must be negatively charged because it has lost the positive charge which the $\mathrm{NH}_{2}$ group has acquired (in being converted to $\mathrm{NH}_{3}$ ). Under these conditions, in the absence of an ionizing solvent, it is natural that the positive and negative groups should be held together electrostatically as indicated by the dotted line in Formula $b$. This formula thus represents clearly the properties of the compound and is fully in accord with the experimental results of Noyes and Potter. Since in this case the oxygen and nitrogen cantnot hold a pair of electrons in common, it seems desirable not to connect them by a valence bond in the formula.

Similar considerations apply to the structure of carbamide.

It is interesting that in the analogous case of the so-called diazophenols studied by Klemenc ${ }^{3}$ the octet theory indicates the possibility of a true ring formula closed by covalence bonds. Klemenc has given evidence for the formula

${ }^{1}$ Chem. Soc. Trans., 107, 715 (1915).

${ }^{2}$ This Journal, 37, 89 (I915).

s Ber., 4\%, 14.07 (1914). 


$$
R \prod_{N=N}^{O}
$$

The octet theory gives $p=5$, which leads to the modification<smiles></smiles>

The covalence of one of the nitrogen atoms is 4 , while that of the other is two, but there is no development of free charges as in the case of the amino acids.

The formula given for diphenylene-diazomethane, $\left(\mathrm{C}_{6} \mathrm{H}_{4}\right)_{2} \mathrm{C}=\mathrm{N} \equiv \mathrm{N}$, ${ }^{1}$ is consistent with the octet theory if the triple bond is replaced by a double bond. In general, it is, perhaps, probable that $>\mathrm{C}=\mathrm{N}=\mathrm{N}$ represents the diazo group rather than<smiles>CC1(C)N=N1</smiles>

The diazonium compounds may have either the structure $(\mathrm{R}-\mathrm{N} \equiv \mathrm{N})^{+}$ $\mathrm{OH}^{-}$or $\mathrm{R}-\mathrm{N}=\mathrm{N}-\mathrm{OH}$. This tautomerism explains the fact that in some cases these compounds exhibit acid as well as alkaline properties, while the unusual triple bond explains the explosive properties. The positive charge is probably not localized on either nitrogen atom but the $-N \equiv N$ group as a whole is positive because it shares its electrons with the $R$ group.

The triazo compounds $R N_{s}$ may have the structure $R-N=N=N$ rather than

$$
\mathrm{R}-\mathrm{N} / \mathrm{N}
$$

Hydronitric acid is then $\mathrm{H}-\mathrm{N}=\mathrm{N}=\mathrm{N}$ and the nitride ion has the very sytrametrical structure $\mathrm{N}=\mathrm{N}=\mathrm{N}$. This structure is in full accord with the experimental facts referred to by Turrentine ${ }^{2}$ in his discussion of the structure of hydronitric acid, from which he concludes that the structure should be $\mathrm{H}-\mathrm{N}=\mathrm{N} \equiv \mathrm{N}$.

'The properties of hydroxylamine indicate that this substance exists in two tautomeric forms ${ }^{3}$ which may be represented according to the octet theory by (I) $\mathrm{H}_{2} \mathrm{~N}-\mathrm{OH}$; and (II) $\mathrm{H}_{3} \mathrm{~N}-\mathrm{O}$; the second of these formulas corresponding to that of the amine oxides already considered. The relationship of this compound to hydrogen peroxide discussed by $\mathrm{I}$. W. Jones is thus made particularly clear, for this latter substance also exists in two tatutometic forms.

a Staudinger and Gatle, Ber., 49, 196r (1916).

${ }^{2}$ This Journal, 36, 23, (1914.)

${ }^{3}$ L. W. Jones, Thus Journal, 36, 1268 (1914). 


\section{(I) $\mathrm{H}-\mathrm{O}-\mathrm{O}-\mathrm{H}$ and (II) $\mathrm{H}_{2} \mathrm{O}-\mathrm{O}$.}

The form II contains in both cases unicovalent oxygen atoms and these formulas thus account directly for the oxidizing properties of these substances.

Hydrogen ions (or nuclei) are able to attach themselves to the free pair of electrons of the nitrogen octet in $\mathrm{H}_{2} \mathrm{NOH}$ to form an ion $\mathrm{H}_{3} \mathrm{NOH}^{+}$. But the tendency to form this ion is weaker than that by which the annmonium ion is formed from ammonia and the hydrogen ion because the larger charge on the nucleus of the oxygen atom displaces the electrons of the nitrogen octet towards the oxygen atom, while the nucleus of the nitrogen atom is displaced in the opposite direction. The presence of the hydroxyl group thus causes a decrease in the force with which the octet of the nitrogen atom holds hydrogen nuclei. There is no necessity for assuming polar valence.

For these reasons hydroxylamine has much weaker basic properties than ammonia. Notwithstanding this, the salts of hydroxylamine such as $\mathrm{NH}_{3} \mathrm{OHCl}$ are completely ionized whether in solution or in the solid state. The reason that ammonia in aqueous solution is a weak base as compared to potassium hydroxide is that only a small fraction of the ammonia molecules combines with hydrogen ions from the water forming ammonium ions and thus liberating hydroxyl ions. The weakness of ammonia as a base is not to be looked upon as due to a lack of ionization of ammonium hydroxide, for this substance, according to the octet theory, can only exist in the completely ionized condition.

\section{Acids, Bases and Salts.}

We have just seen why such substances as ammonia, hydroxylamine, etc., behave as weak bases. The octet theory indicates, however, that potassium hydroxide, barium hydroxide, and tetramethyl ammonium hydroxide can exist only in the ionized condition. That is, even in the solid state, potassium hydroxide consists of potassium ions and hydroxyl ions held together by their electrostatic attraction. In tetramethylammonitum hydroxide, the 4 methyl groups share all four pairs of electrons of the nitrogen octet, so that there are no pairs of electrons by which the oxygen could be held. Here again the substance is completely ionized. It follows, therefore, directly that this substance must be as strong a base as potassium hydroxide. For the same reason the compounds $\mathrm{P}\left(\mathrm{CH}_{3}\right)_{4} \mathrm{OH}$ and $\mathrm{S}\left(\mathrm{CH}_{3}\right)_{3} \mathrm{OH}$ are strong bases.

In the hydrochloric acid molectle the hydrogen nucleus shares a pair of electrons with the chlorine atom. Anhydrous liquid hydrogen chloride is, therefore, a non-conductor of electricity since there are no free ions. But when this substance is brought into contact with water, the hydrogen puclei can attach itself more easily to the unshared pairs of electrons in 
the octets of the water molecules than to the chlorine atoms, since the nuclei of the oxygen atoms have smaller positive charges than those of the chlorine atoms. The result is that the hydrogen nuclei become hydrated hydrogen ions, and the chlorine ions remain in solution. This explanation of the separation of the hydrochloric aeid molecule into ions is not essentially different from that which attributes the effect to the high dielectric constant of the water.

If we consider another substance such as hydrogen sulfide, it is clear that the tendency for the hydrogen nuclei to separate from the octet to which they were originally attached may be very much less. The smaller positive charge on the kernel of the sulfur atom as compared with that of the chlorine atom causes the hydrogen nucleus to be much more firmly held. Another way of expressing this relationship is to say that chlorine readily assumes a form in which it has the covalence of zeto, while sulfur has a greater tendency to be dicovalent than unicovalent. Ammonia, as we have seen, acts as a base rather than as an acid. That it thus tends to take up a fourth hydrogen nucleus instead of giving up those that it has, is due to the smaller positive charge on its kernel, and to the fact that the 4 nuclei enable it to assume a particularly stable form.

For these same reasons methane acts neither as an acid nor as a base. The small charge on the kernel allows the 4 hydrogen nuclei to be very firnly held by the 4 pairs of electrons so that acid properties are absent. There can be no basic properties because the molecule cannot take up any more hydrogen nuclei, all 4 pairs of electrons in the octet already being occupied.

From this viewpoint, acids are substances from whose molectles hydrogen nuclei are readily detached, while bases are substances whose molecules can easily take up hydrogen nuclei. The more easily the hydrogen nuclei are given up, the stronger the acid which results: the greater the tendency to take up hydrogen nuclei the stronger the base. Typical salts, on the other hand, are completely ionized even in the solid state, and are, of course, completely ionized in solution. The variation in electric conductivities, osmotic pressures, etc., of salt solutions is quantitatively explained by Milner and Ghosh as due to the constraints caused by the electric charges which tend to produce a uniform distribution of positive and negative ions, whereas if the particles were uncharged there would be a random distribution.

There are certain substances usually regarded as salts, whose aqueous solutions show relatively little conductivity. Mercuric chloride and cyanide, cadmium iodide, etc., are typical examples of this kind. Mercuric and cadmium ions unquestionably have strong external fields of force in addition to the forces caused by their electric charges. According to the theory developed in a previous paper the ions of calcium and 
strontium differ from atoms of argon and krypton only in their electric charges. Since the stray forces around the atoms of the inert gases are very small, it follows then that the properties of the ions of calcium, strontium, etc., are determined almost wholly by the charges on the ions. But cadmium and mercuric ions have structures like atoms of palladium and platinum, respectively, so that, as in these latter atoms, it must be assumed that there are strong fields of force around the ions in addition to those caused by their charges. It is, therefore, probable that in mercuric chloride, the atoms exist as ions, but that these ions do not separate from each other when the substance goes into solution. The existence of the various organic compounds and complex ions of mercury furnishes abundant confirmation of this theory of strong secondary valence forces about atoms of mercury and other related elements.

Isomerism of Cyanates, Cyanides, Nitrides, Etc.

It is well known that organic cyanates, cyanides and nitrites, exist in two isomeric forms. According to the octet theory the constitutions of these substances are as follows:

\begin{tabular}{|c|c|c|}
\hline Cyanates................... & $\begin{array}{c}\text { Normal } \\
\mathrm{R}-\mathrm{O}=\mathrm{C}=\mathrm{N} \\
\text { or } \mathrm{R}-\mathrm{O}-\mathrm{C} \equiv \mathrm{N}\end{array}$ & $\begin{array}{c}\text { Iso } \\
\mathrm{R}-\mathrm{N}=\mathrm{C}=\mathrm{O}\end{array}$ \\
\hline Cyanides or nitriles.... & $\mathrm{R}-\mathrm{C} \equiv \mathrm{N}$ & $\mathrm{R}-(\mathrm{NC})$ \\
\hline ompounds... & $\mathrm{R}-\mathrm{O}-\mathrm{N}=\mathrm{O}$ & $R \rightarrow 1$ \\
\hline
\end{tabular}

The second column gives $p$ the number of pairs of electrons held in common between the atoms as calculated from the octet equation.

In the isonitriles the nitrogen and carbon kernels are probably contained within a single octet as in the cyanogen ion, and in the nitrogen and carbon. monoxide molecules.

If we apply the octet theory to the structure of inorganic cyanates, cyanides and nitrites we find the following constitutions:

$$
\begin{array}{ll}
\text { Cyanates, } p=4, & \mathrm{~K}+[\mathrm{O}=\mathrm{C}=\mathrm{N}]^{-} \\
\text {Cyanides, } & \mathrm{K}+[\mathrm{CN}]^{-} \\
\text {Nitrites, } p=3, & \mathrm{~K}+[\mathrm{O}-\mathrm{N}=\mathrm{N}]^{-}
\end{array}
$$

The theory thus indicates that these substances differ essentially from the corresponding organic compounds in that the atoms of the metal are not attached to definite atoms of the acid radical. It is, therefore, evident from the octet theory that these substances should not exist in two isomeric modifications, and that the structures of these inorganic compounds do not correspond to either the normal or to the iso organic derivations. These conclusions are in full accord with the facts.

Free cyanic, hydrocyanic and nitrous acids exist in only one form each, although we cannot assume that these exist like their salts, in the com- 
pletely ionized condition. These facts simply illustrate again the mobility of the hydrogen nucleus when bound by atoms having a covalence less than 4. We may, therefore, consider that in these free acids both the modifications observed among the organic derivatives exist together in tautomeric equilibrium.

\section{Phosphonium, Arsonium, Sulfonium and Oxonium Compounds.}

Whereas the ammonia molecule has a relatively strong tendency to take up a hydrogen nucleus to form the ammonium ion, the corresponding tendency of phosphine to take up the hydrogen nucleus is very weak. This decrease in the affinity for hydrogen nuclei in passing from nitrogen to phosphorus, corresponds to the fact that hydrochloric acid is a stronger acid than hydrofluoric and hydrogen sulfide has stronger acid properties than water.

The introduction of a chlorine atom into acetic acid to form chloroacetic acid, according to the viewpoint of $G$. N. Lewis, displaces all the electrons towards the chlorine atom. This decreases the forces holding the hydrogen nucleus in the hydroxyl group and by allowing this hydrogen ion to be more readily detached, makes the chloroacetic acid a stronger acid than acetic. A replacement of the same hydrogen atoms by alkyl radicals allows the electrons to shift towards the hydroxyl group, increasing the forces holding the hydrogen nucleus and making the acid weaker. In a similar way the replacement of the hydrogen atoms in phosphine increases the tendency for the molecule to take up hydrogen nuclei and strengthens the basic properties. Thus trimethyl-phosphine has distinctly basic properties, although these are much less than those of trimethylamine.

On the other hand the phosphonium bases or quaternary compounds such as tetramethyl-phosphonium hydroxide are exceedingly strong bases quite comparable with potassium hydroxide and tetramethyl-ammonium hydroxide. In these compounds, just as in ordinary ammonium salts the central atom must be quadricovalent and the negative radical thus exists as an ion which does not share electrons with the positive radical.

The basic properties of the arseine derivatives are still less marked. Thus even tertiary arsines do not have basic properties. On the other hand, as is to be expected, the quaternary arsonium bases are about as strongly basic as potassium hydroxide and the other quaternary bases.

As distinguished from the corresponding ammonium compounds, it is possible to replace the hydrogen of alkyl substituted phosphines and especially arsines by chlorine. All such compounds whose formulas can be written by giving arsenic a valence of 3 are of course in agreement with the octet theory. The structures of the various chlorine addition compounds, should however, be as follows: 


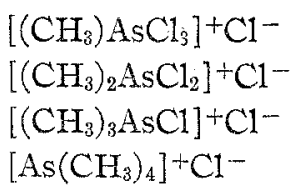

In the corresponding oxides $\left(\mathrm{CH}_{3}\right) \mathrm{AsO}(\mathrm{OH})_{2}, \quad\left(\mathrm{CH}_{3}\right)_{2} \mathrm{AsO}(\mathrm{OH})$ and $\left(\mathrm{CH}_{3}\right)_{3} \mathrm{AsO}$, all 4 radicals are attached directly to the quadricovalent arsenic atom, so that these compounds are not salt-like in character.

For sulfonium compounds such as $\left(\mathrm{CH}_{3}\right)_{3} \mathrm{SI}$ we place $e=34, n=5$ and find $p=3$. The constitution is thus $\left[\mathrm{S}\left(\mathrm{CH}_{3}\right)_{3}\right]^{+} \mathrm{I}^{-}$, where the sulfur atom is tercovalent. The corresponding base $\left.\left[\mathrm{S}_{(\mathrm{CH}}\right)_{3}\right]+\mathrm{OH}-$ is, of course, completely ionized and is, therefore, comparable to potassium hydroxide.

In compounds with oxygen and organic radicals, sulfur takes, with almost equal ease, covalences of 3 or 4 . This leads to a great variety of organic compounds. The following table gives a list of such types of compounds together with the constitutions as derived from the octet theory: Organic Sulfur-Oxygen Compounds, Fitc. Quadricovalent sulfur.

A. Sulfates

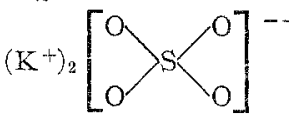

F. Sulfites Tercovalent sulfur.

B. Sulfonates<smiles>[Y]COS1([O])[CH]O1</smiles>

G. Sulfinates

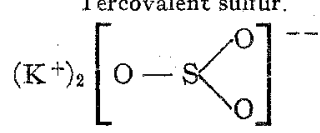

C. Sulfones<smiles></smiles>

H. Dialkyl sulfoxides

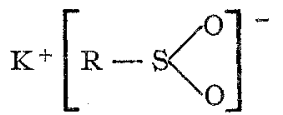

C. Sulfones<smiles>[R][Si]1([R7])CCO1</smiles>

I. Sulfonium compounds $\left[R-S<{ }_{R}^{R}\right]^{+}$

E. Tetralkyl sulfonium iodide<smiles>[R1]S1([R1])CC1</smiles>

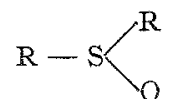

$D$. Trialkyl sulfine iodide $\left[\begin{array}{l}R \\ R\end{array} S\left\langle{ }_{O}^{R}\right]^{+}\right.$ $\left(\mathrm{I}^{-}\right)_{2}$

In addition to these there are, of course, various possible esters of the acids, such, as

A.<smiles>[R20][Si]([R20])(O)O</smiles>

B.<smiles>[R][Si]([R20])(O)O</smiles>

F.<smiles>[R20]S([R20])([R6])O</smiles>
and and<smiles>[Y]C1CC[Ge](O)(O)O1</smiles>

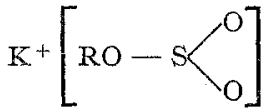


where the letters $A, B, F$ and $G$ refer to the corresponding substances in the preceding table.

The compounds given under $\mathrm{E}$ in the first table, namely, compounds of the type $\left[\mathrm{S}\left(\mathrm{CH}_{3}\right)_{4}\right]^{++} \mathrm{I}_{2}-$ have, I believe, not been isolated. But a closely corresponding cyclic compound trimethylene-dimethyl-sulfonium diiodide $\left[\mathrm{CH}_{2} \cdot \mathrm{CH}_{2} \cdot \mathrm{CH}_{2} \cdot \mathrm{S}\left(\mathrm{CH}_{3}\right)_{2}\right]^{++} \mathrm{I}_{2}-$ has been prepared and studied by

Grishkevich-Trokhimovskii. ${ }^{1}$ He found that both iodine atoms are equally ionized and are precipitated by silver nitrate.

For oxonium compounds such as those of the type $\mathrm{R}_{2} \mathrm{O}$.HX we place $e=28 ; n=4$ and find $p=2$. The hydrogen nucleus can, however, attach itself to one of the unoccupied pairs of electrons of the oxygen atom so we arrive at the structure

$$
\left[\begin{array}{l}
\mathrm{R} \\
\mathrm{R}
\end{array} \mathrm{O}-\mathrm{H}\right]^{+} \mathrm{X}^{-}
$$

For compounds of the $\mathrm{R}_{2} \mathrm{O} \cdot \mathrm{RX}$ type we place $e=34 ; n=5$ and find $p=3$, whence the constitution is

$$
\left[\begin{array}{l}
R \\
R
\end{array} O-R\right]^{+} x^{-}
$$

corresponding exactly to the sulfonium compounds.

The constitutions of the phosphonium, sulfonium, oxonium, compounds, etc., arrived at by the octet theory correspond to those found by Werner's theory. The octet theory, however, has the advantage that it does not require special assumptions regarding valence for compounds of each of these types.

\section{Summary.}

The octet theory of valence which has been described in previous papers is here applied particularly to organic nitrogen compounds, although the general application of the theory to inorganic nitrogen compounds and to salts is discussed.

The numbers of available electrons in the outside shell of any atom is usually given by the ordinal number of the column of the periodic table in which the element is found. This number, which we may represent by $E$, corresponds to the maximum positive valence of the ordinary valence theory and is one for sodium, 4 for carbon, 5 for nitrogen, 6 for oxygen, and 7 for chlorine.

It is readily proved that the octet theory is entirely in agreement with the ordinary valence theory whenever the ordinary formulas are based on valences of unity for hydrogen and $8-E$ for each other element. Thus the ordinary formulas agree with those of the octet theory whenever we

' $J$. Russ. Phys. Chem. Soc., 48, 880 (1916). 
take the following valences: hydrogen, one; carbon, 4 ; nitrogen and phosphorus, 3; oxygen and sulfur, 2; and chlorine, bromine, etc., one. On the other hand all formulas in which valences different from these have been used require modification according to the octet theory.

The octet theory is applied, among others, to the following compounds, whose formulas require modification.

Sodium chloride $\mathrm{Na}^{+} \mathrm{Cl}^{-}$(the covalence of both atoms is zero).

Ammonium chloride<smiles>CCOCCOCCOCC</smiles>

Triphenylmethyltetramethyl ammonium $\left[\mathrm{N}\left(\mathrm{CH}_{8}\right)_{4}\right]^{+}\left[\mathrm{CPh}_{3}\right]^{-}$(the nitrogen is quadricovalent while the central carbon atom in the anion is tercovalent).

Diazophenol<smiles>[R1]N1CO1</smiles>

Diazonium compounds $[\mathrm{R}-\mathrm{N} \equiv \mathrm{N}]+\mathrm{OH}-$ or $\mathrm{R}-\mathrm{N}=\mathrm{NOH}$

Triazo-compounds $\mathrm{R}-\mathrm{N}=\mathrm{N}=\mathrm{N}$

Hydroxylamine $\mathrm{H}_{2} \mathrm{NOH}$ or $\mathrm{H}_{8} \mathrm{~N}-\mathrm{O}$, etc.

According to this theory all salts are completely ionized even before they are brought into solution. This conclusion is, however, in accord with recent work of Milner, Ghosh and others. It explains why there are weak acids and weak bases but no weak salts.

The known cases of isomerism, including stereoisomerism, of nitrogen, phosphorus and sulfur compounds are in full accord with the octet theory.

The fact that organic cyanates, cyanides and nitrites exist in two isomeric forms, while the corresponding inorganic salts exist in only one form, is explained, since the nulcovalent atoms of the metals in the inorganic compounds are not attached to definite atoms of the acid radicals.

The available data on phosphonium, arsonium, sulfonium and oxonium compounds are in full accord with the octet theory, which gives for these compounds constitutions closely resembling those previously given by Werner.

SCHENECTADY, N. X. 